\title{
Erratum to: Geometry Behind Chordal Loewner Chains
}

\author{
Manuel D. Contreras • Santiago Díaz-Madrigal • \\ Pavel Gumenyuk
}

\section{Erratum to: Complex Anal. Oper. Theory (2010) 4:541-587 DOI 10.1007/s11785-010-0057-6}

The authors apologize for several mistakes and misprints found after the paper "Geometry behind chordal Loewner chains" was published. The following corrections are necessary in Lemmas 2.1, 2.2 and 3.6.

\section{Lemma 2.1 should be as follows:}

Lemma 2.1 Let $\varphi$ be a holomorphic univalent self-mapping of the unit disk $\mathbb{D}$ with $\varphi(0)=0$. Then:

(1) for all $z \in \mathbb{D}$,

$$
|\varphi(z)-z| \leq\left|1-\varphi^{\prime}(0)\right|+\frac{|z|^{2}}{\sqrt{1-|z|^{2}}} \sqrt{1-\left|\varphi^{\prime}(0)\right|^{2}}
$$

The online version of the original article can be found under doi:10.1007/s11785-010-0057-6.

M. D. Contreras $(\varangle) \cdot$ S. Díaz-Madrigal

Camino de los Descubrimientos, s/n, Departamento de Matemática Aplicada II,

Escuela Técnica Superior de Ingenieros, Universidad de Sevilla, Sevilla 41092, Spain

e-mail: contreras@us.es

S. Díaz-Madrigal

e-mail: madrigal@us.es

P. Gumenyuk

Department of Mathematics, University of Bergen, Johannes Brunsgate 12, Bergen 5008, Norway e-mail: Pavel.Gumenyuk@math.uib.no 
(2) if $\varphi^{\prime}(0)>0$, then for all $z \in \mathbb{D}$,

$$
|\varphi(z)-z| \leq C_{0} \sqrt{1-\varphi^{\prime}(0)}
$$

where $C_{0}:=1+\frac{\sqrt{2} r^{2}}{\sqrt{1-r^{2}}}$ and $r:=|z|$.

Proof Write $\varphi(z)=\sum_{n=1}^{\infty} a_{n} z^{n}$. Let us recall that

$$
\sum_{n=1}^{\infty} n\left|a_{n}\right|^{2}=m(\varphi(\mathbb{D})) \leq m(\mathbb{D})=1
$$

where $m(\cdot)$ stands for the normalized Lebesgue measure in the unit disc. Let $z \in \mathbb{D}$ and $r:=|z|$. Then

$$
\begin{aligned}
& |\varphi(z)-z| \leq\left|\sum_{n=2}^{\infty} a_{n} z^{n}\right|+\left|1-\varphi^{\prime}(0)\right| \leq \sqrt{\sum_{n=2}^{\infty} r^{2 n}} \sqrt{\sum_{n=2}^{\infty}\left|a_{n}\right|^{2}}+\left|1-\varphi^{\prime}(0)\right| \\
& =\frac{r^{2}}{\sqrt{1-r^{2}}} \sqrt{\sum_{n=2}^{\infty}\left|a_{n}\right|^{2}}+\left|1-\varphi^{\prime}(0)\right| \leq \frac{r^{2}}{\sqrt{1-r^{2}}} \sqrt{\sum_{n=2}^{\infty} n\left|a_{n}\right|^{2}}+\left|1-\varphi^{\prime}(0)\right| \\
& \leq \frac{r^{2}}{\sqrt{1-r^{2}}} \sqrt{1-\left|\varphi^{\prime}(0)\right|^{2}}+\left|1-\varphi^{\prime}(0)\right| \text {. }
\end{aligned}
$$

This proves (1). Assertion (2) is now an immediate consequence of (1).

\section{The last line in the proof of Lemma 2.2 has to be changed as follows:}

$$
|f(z)-g(z)| \leq g^{\prime}(0) \frac{2 C_{0}}{(1-r)^{3}} \sqrt{1-\varphi^{\prime}(0)}=\frac{2 C_{0}}{(1-r)^{3}} \sqrt{g^{\prime}(0)\left(g^{\prime}(0)-f^{\prime}(0)\right)} .
$$

III. The proof of Lemma 3.6 contains an error. Arguing in a more direct way we get a better estimate under weaker conditions $|z|>b>0$ and $|\arg z-\pi / 2| \leq \pi / 3$ :

$$
\begin{aligned}
& \frac{1+r_{\mathbb{H}}(z, i b)}{1-r_{\mathbb{H}}(z, i b)}=\frac{|z+i b|+|z-i b|}{|z+i b|-|z-i b|}=\frac{(|z+i b|+|z-i b|)^{2}}{|z+i b|^{2}-|z-i b|^{2}} \\
& =\frac{(|z+i b|+|z-i b|)^{2}}{4 b \operatorname{lm} z} \leq \frac{(2|z|+2 b)^{2}}{4 b \cdot \frac{1}{2}|z|} \leq \frac{2}{b}|z| .
\end{aligned}
$$

\section{Misprints:}

1. Condition (B.2) in Theorem 4.8 should contain " $\Omega_{t}$ " instead of " $\Omega_{t}(\mathbb{D})$ ".

2. The estimate of $|V(z, x) / z|$ in the proof of Proposition 5.9, see page 572, should contain " $|\mathrm{Re} z|$ " instead of "Re $z$ ".

3. The Eq. (7.5) on p. 585 should contain the formula $u(t):=H^{-1}(\lambda(t)) \neq 1$ rather than $u(t):=H(\lambda(t)) \neq 1$. 